\title{
Panorama imaging for image-to- physical registration of narrow drill holes inside spongy bones
}

Jan Bergmeier, Jacob Friedemann Fast, Tobias Ortmaier, Lüder Alexander Kahrs

Jan Bergmeier, Jacob Friedemann Fast, Tobias Ortmaier, Lüder Alexander Kahrs, "Panorama imaging for image-to-physical registration of narrow drill holes inside spongy bones," Proc. SPIE 10135, Medical Imaging 2017: Image-Guided Procedures, Robotic Interventions, and Modeling, 1013507 (3 March 2017); doi: 10.1117/12.2254417 


\title{
Panorama imaging for image-to-physical registration of narrow drill holes inside spongy bones
}

\author{
Jan Bergmeier, Jacob Friedemann Fast, Tobias Ortmaier, Lüder Alexander Kahrs \\ Gottfried Wilhelm Leibniz Universität Hannover, Faculty of Mechanical Engineering, \\ Institute of Mechatronic Systems, Appelstr. 11a, 30167 Hannover, Germany
}

\begin{abstract}
Image-to-physical registration based on volumetric data like computed tomography on the one side and intraoperative endoscopic images on the other side is an important method for various surgical applications. In this contribution, we present methods to generate panoramic views from endoscopic recordings for image-to-physical registration of narrow drill holes inside spongy bone. One core application is the registration of drill poses inside the mastoid during minimally invasive cochlear implantations. Besides the development of image processing software for registration, investigations are performed on a miniaturized optical system, achieving $360^{\circ}$ radial imaging with one shot by extending a conventional, small, rigid, rod lens endoscope. A reflective cone geometry is used to deflect radially incoming light rays into the endoscope optics. Therefore, a cone mirror is mounted in front of a conventional $0^{\circ}$ endoscope. Furthermore, panoramic images of inner drill hole surfaces in artificial bone material are created. Prior to drilling, cone beam computed tomography data is acquired from this artificial bone and simulated endoscopic views are generated from this data. A qualitative and quantitative image comparison of resulting views in terms of image-to-image registration is performed. First results show that downsizing of panoramic optics to a diameter of $3 \mathrm{~mm}$ is possible. Conventional rigid rod lens endoscopes can be extended to produce suitable panoramic one-shot image data. Using unrolling and stitching methods, images of the inner drill hole surface similar to computed tomography image data of the same surface were created. Registration is performed on ten perturbations of the search space and results in target registration errors of $(0.487 \pm 0.438) \mathrm{mm}$ at the entry point and $(0.957 \pm 0.948) \mathrm{mm}$ at the exit as well as an angular error of $(1.763 \pm 1.536)^{\circ}$. The results show suitability of this image data for image-to-image registration. Analysis of the error components in different directions reveals a strong influence of the pattern structure, meaning higher diversity results into smaller errors.
\end{abstract}

Keywords: image-guided surgery, intraoperative imaging, endoscopic procedures, cochlear implantation

\section{INTRODUCTION}

Endoscopy is a widespread technique for imaging cavities of the human body and hidden regions without a direct line of sight. Minimization has resulted in diameters of commercially available rod lens endoscopes as low as $1.9 \mathrm{~mm}$. Available optics provide either a straight or an oblique viewing angle with respect to the endoscope shaft axis. Both versions come with drawbacks for certain applications.

A new registration method based on endoscopy of drill holes in unique bone structures, e.g. mastoid cells in the temporal bone, was recently presented. ${ }^{1,2}$ One core application is the verification of the drill pose during minimally invasive cochlear implantations. ${ }^{3-5}$ Since drilling is performed in highly sensitive areas inside the human head during these operations - crucial structures like the facial nerve, chorda tympani etc. can be damaged - research is highly advisable to ensure atraumatic drilling precision control. ${ }^{6}$ In preparation of a minimally invasive electrode insertion procedure, the drilling trajectory passes through the temporal bone, more precisely the mastoid process. This anatomical structure contains numerous air pockets - the so-called mastoid cells - that form an unique pattern. During drilling, a cylindrical cavity is cut into this bone structure.

The proposed method employs a small, commercial endoscope to inspect the surface of drill holes after pilot drilling of some $\mathrm{mm}$ depth has been performed. Comparison of the recorded surface structure with preoperative

Address all correspondence to: Jan Bergmeier, E-mail: jan.bergmeier@imes.uni-hannover.de,

Tel: +49 (0)511-762-17839, Fax: +49 (0)511-762-19976

Medical Imaging 2017: Image-Guided Procedures, Robotic Interventions, and Modeling, edited by

Robert J. Webster III, Baowei Fei, Proc. of SPIE Vol. 10135, 1013507 · @ 2017 SPIE

CCC code: $1605-7422 / 17 / \$ 18 \cdot$ doi: $10.1117 / 12.2254417$

Proc. of SPIE Vol. 10135 1013507-1 
image data, obtained by modalities such as computed tomography (CT) or magnetic resonance imaging (MRI), enables intraoperative identification of the drill hole pose. Based on the knowledge of the actual pilot drilling position, the surgeon is able to decide whether the intervention can be pursued as planned.

The first step of this registration method consists of cutting out and unrolling the tubular drill hole surface from the preoperative image data to obtain a planar image, as shown in Fig. 1 a). Calculating these unrolled drill hole surfaces from preoperative image data for multiple drill hole poses yields a series of images that is compared to the unrolled endoscopic image. Besides high-resolution preoperative imaging, convenient, accurate and fast image acquisition techniques are crucial for the endoscopic part of the registration method.

Since the minimally invasive procedure demands small drill diameters, limited to approximately $4 \mathrm{~mm}$, size constraints for the medical endoscope are important. Commercial endoscopes with a diameter of $1.9 \mathrm{~mm}$ are available in a straight-viewing variant (viewing angle $0^{\circ}$ ) or several oblique-viewing versions (e.g. with a viewing angle of $70^{\circ}$ or $90^{\circ}$ ). Whenever oblique-viewing endoscopes are employed at extremely short working distances, only a small area of the drill hole surface can be explored with a single image. The more image data can be gathered, the more features can be accessed to perform inter-modal registration. Therefore, the optimum procedure would be to record a $360^{\circ}$ view over the full length of the drill hole. In order to achieve this, obliqueviewing endoscopes need to be turned around the shaft axis while performing a feed motion of the optical device, which means a time consuming image acquisition.

Bearing in mind the limited field of view of available endoscopes and the small working distance in narrow drillings, an imaging solution enlarging the field of view while limiting distortion and offering a short focal length might improve inspection of mastoid cavities. Currently available systems, mainly for industrial applications, offer an enlarged endoscopic field of view by using different solutions such as panorama lenses, camera arrays, and other. While showing advantages and disadvantages, all solutions known to the authors share an insufficient degree of miniaturization and can therefore not be applied to minimally invasive medical applications. Thus, we consider miniaturization of panoramic optics for medical endoscopy to be an open topic in research and development.

In Fig. 1 b), a straight-viewing (left), oblique-viewing (middle) and panoramic cone endoscope system (right) are compared with respect to their capacity of imaging structures on narrow drill walls. While the straightviewing optics allows for partial inspection of cavities arranged in a circular region in front of the distal endoscope end, the oblique-viewing assembly offers slightly more profound examination of cavities located in its direction of view. The panoramic cone optics on the right allows thorough inspection of the deeper drill wall layers. Straight-viewing endoscopes provide a tunnel vision image - no image information is gathered perpendicularly to the endoscope axis. Furthermore, the resolution of the drill hole wall image decreases with increasing distance to the endoscope. Moreover, only the inner mastoid cell surface that lies in viewing direction is visible - the surface structure against that direction can not be imaged. This illustrates that the recording of the mastoid structure depends on the imaging direction when using straight-viewing endoscope systems. Direction-dependent effects are not considered when calculating the drill hole surface from preoperative image data, thus making them undesirable.
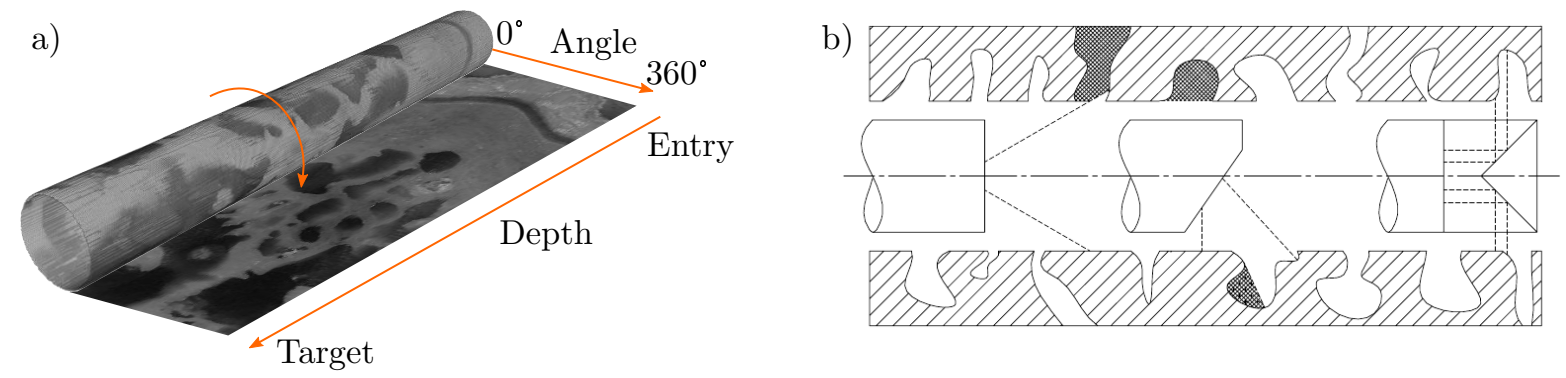

Figure 1. Drill hole surface inside the mastoid and comparison of endoscope optics. a) Drill hole surface of a trajectory inside the mastoid showing the bone-air pattern. Next to the cylinder, the surface is unrolled for better visibility of the pattern and registration purposes. b) Comparison of straight-viewing (left), oblique-viewing (middle) and panoramic cone endoscope system (right) performance. Blackened areas show shadowed areas that can not imaged by the respective endoscope optics. 
The development of a new optical system is only the first step to acquire panoramic images of the tubular surface in narrow drill holes. Another important factor is subsequent image processing, transforming circular image frames into planar, unrolled images and creating a large image of the tube surface from several video frames, using stitching techniques. ${ }^{7}$ Employing a cone mirror drastically reduces the effort to achieve these images, since the optics records the surface perpendicularly to the optical axis of the endoscope. Also, endoscopic images that show a higher degree of similarity with respect to the image data extracted at the drill hole surface from the CT image are created. Still, several image processing steps are required to unfold the image data and produce a connected image from several frames along the insertion direction.

\subsection{Related work}

Multiple solutions for providing panoramic images with a large field of view, combining endoscopes with special optics or using custom-built imaging devices, have been developed. Numerous patents indicate sustained research activity in this area. Solutions include fish-eye optics, camera arrays and several other approaches. In this context, a patent filed in 1978 by Richard Wolf GmbH (Knittlingen, Germany) is of special interest. ${ }^{8}$ It reveals a panoramic optical system consisting of a cone recess in a glass cylinder. However, spatial dimensions as well as manufacturing techniques are not further specified. To the best of our knowledge, no endoscope based on this patent is available for clinical use.

Ou-Yang et al. ${ }^{9}$ have demonstrated that a cone mirror is well suited for radial imaging of tubular structures. They have designed a capsule endoscope system called Radial Imaging Capsule Endoscope (RICE) that makes use of a conical mirror featuring a cone angle of $90^{\circ}$ and a radius of $3.35 \mathrm{~mm}$ to reflect radial light rays onto the CCD sensor located at the center of the capsule and directly facing the cone mirror. Developed for gastroenterological applications, it has an outer diameter of $10 \mathrm{~mm}$. One advantage of this approach in comparison with spherical lenses is the reduced image processing effort to obtain undistorted image data. A drawback is the high complexity of the image reconstruction procedure as the endoscope system rotates about all three axes during its journey through the body. Experiments carried out on a living animal showed that automatic image stitching using established algorithms lead to some misalignment of the image layers. Furthermore, illumination differences due to the properties of the integrated LED-based illumination system make part of the image data unsuitable for stitching purposes. The problem of inhomogeneous illumination distribution in this assembly is addressed via ray tracing. ${ }^{10}$ The authors conclude that coating the protective cap of the optical system and inclining the annular LEDs by an angle of $-5^{\circ}$ yields optimum image quality results. It should be noted that the RICE system is not designed to be part of an inter-modal image registration procedure. The use of this endoscope capsule is aimed at generating a true color representation of cavities like the bowel that helps physicians find a diagnosis by direct inspection of the image data.

Optical systems associating mirrors are also known from the field of autonomous mobile robotic systems. For example, such a catadioptric system and ways of de-warping gathered image data has been developed by Spaceck et al. ${ }^{11}$

\section{METHODS}

We developed an optical system for surgical endoscopic panorama imaging in narrow drill holes that extends a conventional, rigid $0^{\circ}$ Hopkins II Telescope (KARL STORZ GmbH \& Co. KG, Tuttlingen, Germany) with a shaft diameter of $1.9 \mathrm{~mm}$. Core component is a cone, coated with aluminum to deflect radially incoming light rays into the endoscope optics.

Several concepts that are capable of one-shot $360^{\circ}$ surround imaging in narrow drill holes have been developed and evaluated. After identification of the most promising assembly, we manufactured a prototype device, combined it with the conventional endoscope and recorded first panoramic sequences in artificial bone material. A suitable image processing algorithm was developed to rectify the frames, let the user choose a region of interest to be evaluated, unroll the circular endoscopic images into planar, stripe-shaped frames and combine the resulting frames to produce a picture showing the inner drill hole surface of the whole length that has been explored. This drill hole image is applied to an image-to-image registration method to find the drill hole, most similar to the recorded one from a set of simulated drill hole images. Registration errors are then evaluated by using the measured drill hole pose as reference. 


\subsection{Development of the optical concept}

The target was to obtain a powerful, yet cost-effective optical system for panoramic imaging inside narrow bone drill holes by extending a conventional rod-lens endoscope with a custom assembly. Table 1 shows three concepts that have been researched and compared with regard to their complexity, expected image quality, fitness for clinical applications and manufacturing options.

Table 1. Comparative representation of endoscopic panorama concepts.

\begin{tabular}{|c|c|c|c|}
\hline \multirow[b]{2}{*}{ Properties } & \multicolumn{3}{|c|}{ Concept } \\
\hline & & & \\
\hline Description & Wide angle lens system & Micro camera system & Cone mirror system \\
\hline \multirow[t]{2}{*}{ Imaging quality } & Optical distortion & Low image resolution & Loss of illumination \\
\hline & $\begin{array}{l}\text { Inhomogeneous illumina- } \\
\text { tion }\end{array}$ & $\begin{array}{l}\text { External illumination nec- } \\
\text { essary }\end{array}$ & $\begin{array}{l}\text { Inhomogeneous illumina- } \\
\text { tion }\end{array}$ \\
\hline \multirow[t]{2}{*}{ Complexity } & $\begin{array}{l}\text { Complex lens demands for } \\
\text { complex methods as ray } \\
\text { tracing }\end{array}$ & $\begin{array}{l}\text { Microelectronic } \\
\text { bly with high degree of } \\
\text { miniaturization }\end{array}$ & Simple geometry \\
\hline & Two components & Three components & Three components \\
\hline Manufacturing & $\begin{array}{l}\text { Monolithic manufacturing } \\
\text { from PMMA possible } \\
\text { Manufacturing from quartz } \\
\text { glass possible }\end{array}$ & $\begin{array}{l}\text { Micro camera must be pur- } \\
\text { chased } \\
\text { Protective cap made from } \\
\text { PMMA or quartz glass }\end{array}$ & $\begin{array}{l}\text { At least two raw materials } \\
\text { necessary } \\
\text { (PMMA for cone and hous- } \\
\text { ing, aluminum for coating) }\end{array}$ \\
\hline Costs & $\begin{array}{l}\text { Single-use with low manu- } \\
\text { facturing costs possible }\end{array}$ & High manufacturing costs & High manufacturing costs \\
\hline
\end{tabular}

The wide angle lens concept, described in the left column of Table 1, was discarded due to a potentially high optical distortion and the cost of a custom lens development process. The system shown in the middle of the table, featuring a micro camera, contains a complex electronic assembly that is prone to manufacturing difficulties. By replacing the rod lens endoscope optics with a camera, the good image quality of the conventional Hopkins endoscope cannot be used. Thus, we decided to manufacture a prototype of the system shown in the right column of Table 1 . The simple geometry of the cone mirror used to deflect light rays, originating from the explored drilling wall into the endoscope, reduces the development and manufacturing costs while offering a true one-shot $360^{\circ}$ view of a small section of a drill hole.

\subsection{Prototype assembly}

The intrinsic parameters of the rod lens endoscope were determined experimentally based on the well-established pinhole camera model prior to the development process. To offer internal illumination, the endoscope contains a high number of optical fibers that are arranged asymmetrically around the physical shaft axis (see Fig. 2 a)). Using a SteREO Discovery.V8 stereo microscope (Carl Zeiss Microscopy GmbH, Jena, Germany), a magnified view of the distal endoscope end was obtained to assess the eccentricity of the rod lenses with regard to the endoscope shaft axis. The measurement yielded a value of only $0.08 \mathrm{~mm}$, which is less than $5 \%$ of the overall endoscope diameter. Furthermore, the endoscope's optical opening angle was evaluated by adjusting a circular crosshair-like pattern with known diameter $d$ in the field of view until perfect congruence of the crosshair circle and the circular endoscopic image was achieved. The distance $l$ between the distal endoscope shaft end and the pattern plane was measured using a coordinate measuring machine (Gage Standard, FARO Europe GmbH \& Co. KG, Korntal-Münchigen, Germany). Based on the experimental results, the opening angle $\theta$ was calculated to

$$
\theta=2 \arctan \left(\frac{d}{2} l\right) \approx 60.5^{\circ} .
$$


Thus, to be entirely observable by the Hopkins endoscope, an object with a maximum diameter $b$ should be placed at a minimum viewing distance $l_{\min }$ of

$$
l_{\min }=0.5 b \tan ^{-1}\left(\frac{\theta}{2}\right) \approx 0.857 b .
$$

In order to mount an aluminum-coated cone mirror (Edmund Optics GmbH, Karlsruhe, Germany) with a diameter of $2 \mathrm{~mm}$ and a cone point angle of $90^{\circ}$ in front of the rod lens endoscope without obscuring any radially incoming light rays, a transparent PMMA pipe covering the endoscope shaft as well as the cone mirror was employed. The PMMA pipe has a diameter of $3 \mathrm{~mm}$ and a wall thickness of $0.5 \mathrm{~mm}$. The complete surround imaging system containing the cone mirror and a PMMA protection cap is shown in Fig. 2 b).

a)

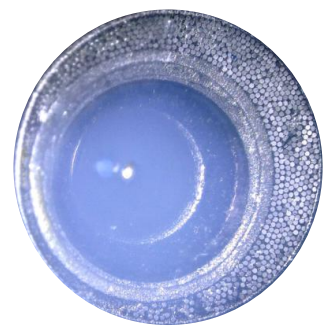

b)

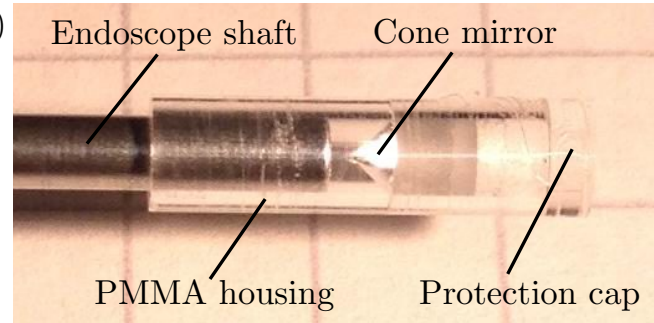

Figure 2. a) Detail view of slightly asymmetric configuration of rod lens array on distal end of endoscope. b) Assembly of the panorama optics and setup. A PMMA housing mounts the cone mirror in front of the distal end of the endoscope shaft.

\subsection{Experimental Setup}

Recording of air pockets in drill holes and processing of the image data was evaluated using a biomechanical test block (Sawbones Europe, Malmö, Sweden) as bone substitute. Similar to Kobler et al., ${ }^{12}$ drill holes simulate mastoid cells, further referred to as mastoid drill holes. Fig. 3 a) gives a detailed view as wire frame representation of the test block. An arbitrary pattern of through holes were drilled into the test block, perpendicular to the intended drill trajectory (red dashed line). The coordinate origin $\mathrm{KS}_{\mathrm{Bl}}$ serves as reference for the block's position throughout all figures. The mastoid drill holes are oriented in x-direction in the block coordinate system.

Our experimental test setup is shown in Fig. 3 b). The Sawbones block on the left is placed on a linear translation stage (MT1-Z8, Thorlabs Inc., Newton, New Jersey, USA), which provides linear movement at a constant feed rate while the endoscope remains fixed on the optical breadboard. The endoscope on the right side is coupled to a zoom lens (Richard Wolf GmbH, Knittlingen, Germany) and a CCD camera (uEye UI-2250-SEC-HQ, IDS Imaging Development Systems GmbH, Obersulm, Deutschland), while the cone optics is attached to the distal endoscope tip.

Fig. 3 c) compares the $0^{\circ}$ view into the drill hole inside mastoid bone to a comparable view inside our test block. Both images reveal mastoid cells that are intersected by the drill hole. While the drill hole surface in the test block is smoother, it creates are more inhomogeneous illumination. In both image, the intersections of the drill hole with the mastoid are clearly visible.

\subsection{Endoscopic image processing}

We attached the cone mirror assembly to the rod lens endoscope and inserted the system into a drill hole at a constant feed rate. Simultaneously, a video sequence was recorded to obtain a series of consecutive frames of the cone mirror surface while exploring the drilling. An exemplary endoscopic view of the cone mirror inside a drill hole in artificial bone material is provided in Fig. 4 a).

Prior to any processing of the obtained image data, the frames of the original sequence are rectified using standard camera calibration methods, customized for endoscopic optics. All rectified frames are then unrolled into a stripe-shaped representation of a drill wall section. The unrolling procedure is illustrated in Fig. $4 \mathrm{~b}$ ). We established an interactive procedure to let the user choose an annular region of interest (ROI) in the endoscopic 

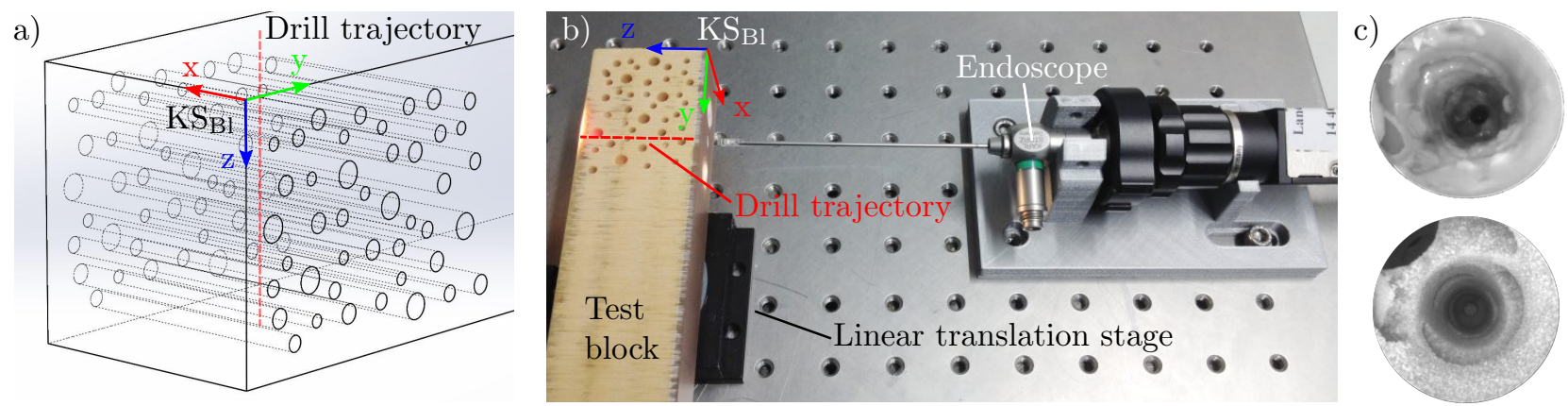

Figure 3. Experimental setup. a) Wire frame representation of biomechanical test block, showing the artificial mastoid cells inside the block. b) Endoscope optics, mounted onto an optical breadboard. The artificial bone block is placed onto a linear translation stage, providing constant feed rate. c) Recording of a $4 \mathrm{~mm}$ drill hole, using a straight-viewing endoscope. Top: Drill hole inside an ex-vivo human temporal bone. Bottom: Drill hole in artificial bone.

image of the cone surface. This ROI is exemplary marked by colored lines in Fig. 4 b) and defined by the radii $r_{1}$ and $r_{2}$. The pixel rings between those radii are unrolled and arranged in horizontal lines to yield the stripe-like representation on the right of Fig. $4 \mathrm{~b}$ ). The colored lines in both pictures possess the same length. A transformation from polar to Cartesian coordinates rearranges the original image pixels to form a stripe-shaped image.

a)

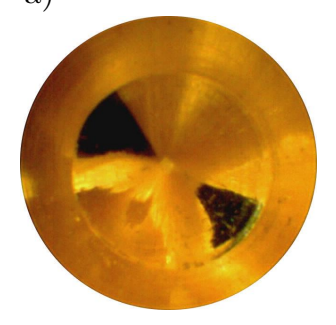

b)

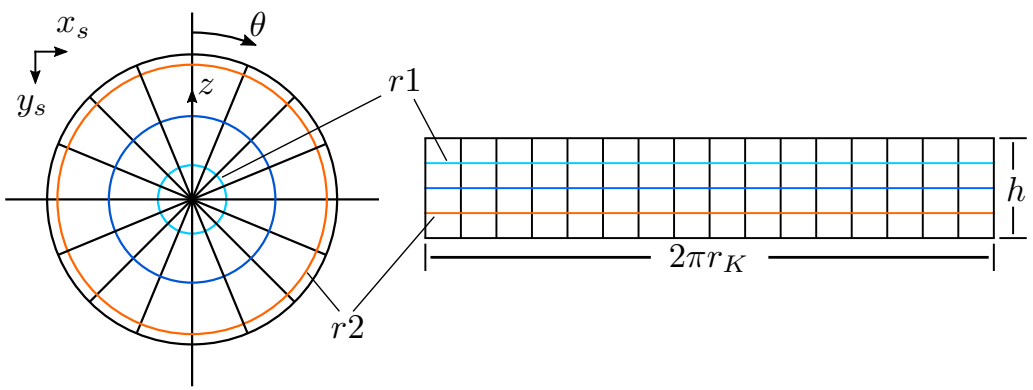

Figure 4. Endoscopic recordings inside drill holes in artificial bone and unrolling procedure. A transformation from polar to Cartesian coordinates is performed. a) Recording using a cone mirror as extension to the $0^{\circ}$ endoscope. b) Scheme of circular recording in polar coordinates $(\theta, z)$ and scheme of planar unrolled recording in Cartesian coordinates $(x, y)$. Colored lines represent corresponding lines in the two image representations.

Stitching of the stripe-shaped frames is achieved using a straightforward method based on the known constant translation velocity of the optical system and the known cone and drill hole geometry. The employed algorithm yields a complete, planar view of the explored drilling that is intended to be used for inter-modal registration with computer tomography datasets.

\subsection{Registration workflow and evaluation}

As proof-of-concept, the intended registration workflow is performed with our developed endoscope optics and biomechanical test block that provides an unique pattern for patient-to-image registration.

Preoperatively, the test block is scanned with a medical CBCT imaging device (xCAT, Xoran Technologies, LLC, MI, USA). The block is then measured with the coordinate measuring machine (FARO, see Sec. 2.1). Three surfaces are recorded and intersected to obtain an origin for a coordinate system $\mathrm{KS}_{\mathrm{Bl}}$ in the corner of the block, yielding the transformation ${ }^{\mathrm{F}} \boldsymbol{T}_{\mathrm{Bl}, 1}$ (see Fig. 3 and Fig. 5). The surface normals are used to define the coordinate axes. Analogous, the block origin in image coordinates $\mathrm{KS}_{\mathrm{I}}$ of the CT image data is determined by manual user input to obtain the transformation ${ }^{\mathrm{I}} \boldsymbol{T}_{\mathrm{Bl}, 2}$. Three edges as well as the origin are clicked by the user and used for calculation of the block's origin and coordinate axes. Chaining up the coordinate transformations enables calculation of measured points in image coordinates. 
Fig. 5 shows all the coordinate systems and transformations. Block coordinate systems in FARO and image coordinates are marked with $\mathrm{Bl}, 1$ and $\mathrm{Bl}, 2$, respective. This demonstrates, that both do not necessarily have the same orientation and position relative to the block, due to registration inaccuracies.

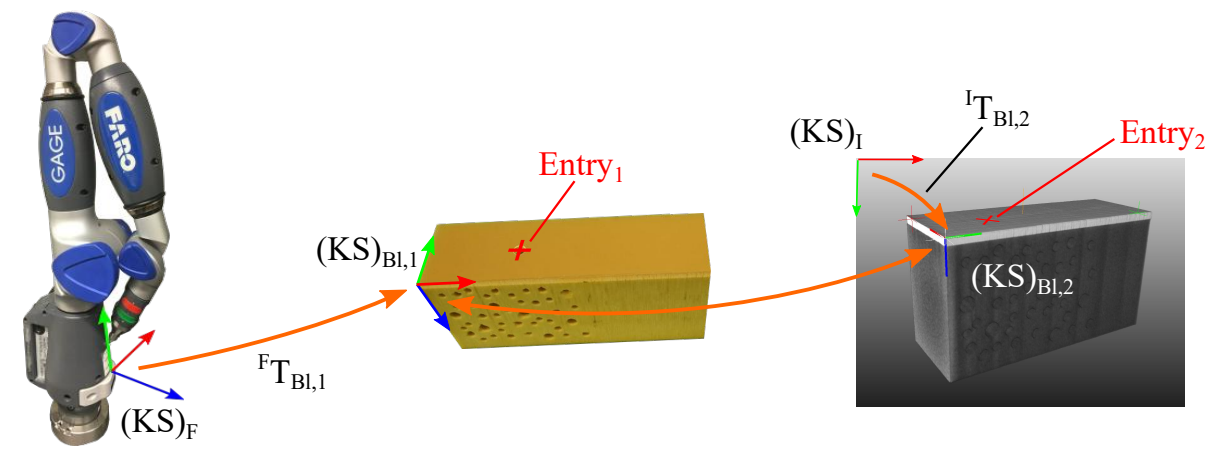

Figure 5. Transformations are coordinate systems for the coordinate measuring machine, the test block and the volumetric image data.

The next step is drilling a $4 \mathrm{~mm}$ hole from the top to the bottom (z-direction in $\mathrm{KS}_{\mathrm{Bl}}$ ) into the test block. Measured entry and exit points of the drill hole in the block that define the drill hole pose are transformed into image coordinates and used for the search space definition. To simulate drilling inaccuracies, the measured trajectory is perturbed with an arbitrary offset. This 'perturbed' search space is then applied to the drill image calculation from the preoperative volumetric image data. Ten perturbed search spaces are created. More details about the search space definition and drill image calculation can be found in our former publication. ${ }^{1}$

An overview of the registration workflow is given in Fig. 6. Endoscopic image data from the inner drill hole surface is created using the previously described methods. The unrolled and stitched endoscopic panorama image of the full length of the drill hole serves as floating image for our registration. A registration approach based on the edges of the visible mastoid cells has been chosen for image-to-image comparison. Edges are detected using Gauss smoothing, a Sobel edge detection filter, a binary image filter and a connected components labeling, removing regions with small components and therefore 'cleaning' the edge image. For the unrolled CT drill hole images, it is sufficient to use the edge detection and binary image filters.

Image-to-image comparison is performed employing a chamfer matching method ${ }^{13}$ that compares the edge image to a distance map. For each double unrolled CT image, a distance map image, using a signed Maurer distance map filter, ${ }^{14}$ is calculated. The endoscopic edge image is then registered to the CT distance map by summing up all values that are overlayed by the mastoid edges in the endoscopic image. The best result serves as measure for the similarity of the endoscopic to the CT drill hole image.

In the last step, errors between the registered and the measured trajectory are evaluated. The target registration error (TRE) is calculated at entry and exit point as well as in a depth of $30 \mathrm{~mm}$, which is approximately the depth of the facial recess in a human head, measured from the skull surface. Each TRE error is resolved into $\mathrm{x}$ - and $\mathrm{y}$-components in block coordinates. While the $\mathrm{x}$-coordinate represents the direction of the mastoid drill holes, the y-direction represents the orthogonal direction (compare to Fig. 3 a)). Further, the angular deviation that lies between both trajectories is determined.

\section{RESULTS}

Fig. 7 shows unrolled and stitched images of a drill hole surface, created from endoscopic and CT image data. A drill hole with diameter of $4 \mathrm{~mm}$ intersects artificial bone and simulated mastoid cavities inside the Sawbones block and thus produces the pattern, presented in the three images.

Fig. 7 a) shows the stitching result using consecutive image frames. A drill hole with a diameter of $4 \mathrm{~mm}$ inside the Sawbone sample block was examined with the $0^{\circ}$ Storz Hopkins II endoscope system. Image frames, like in Fig. 3 c) (bottom), are unrolled similar to Sec. 2.4 by determining the image center and performing a coordinate transformation from polar to Cartesian coordinates. Stitching was performed applying Image Composite Editor 


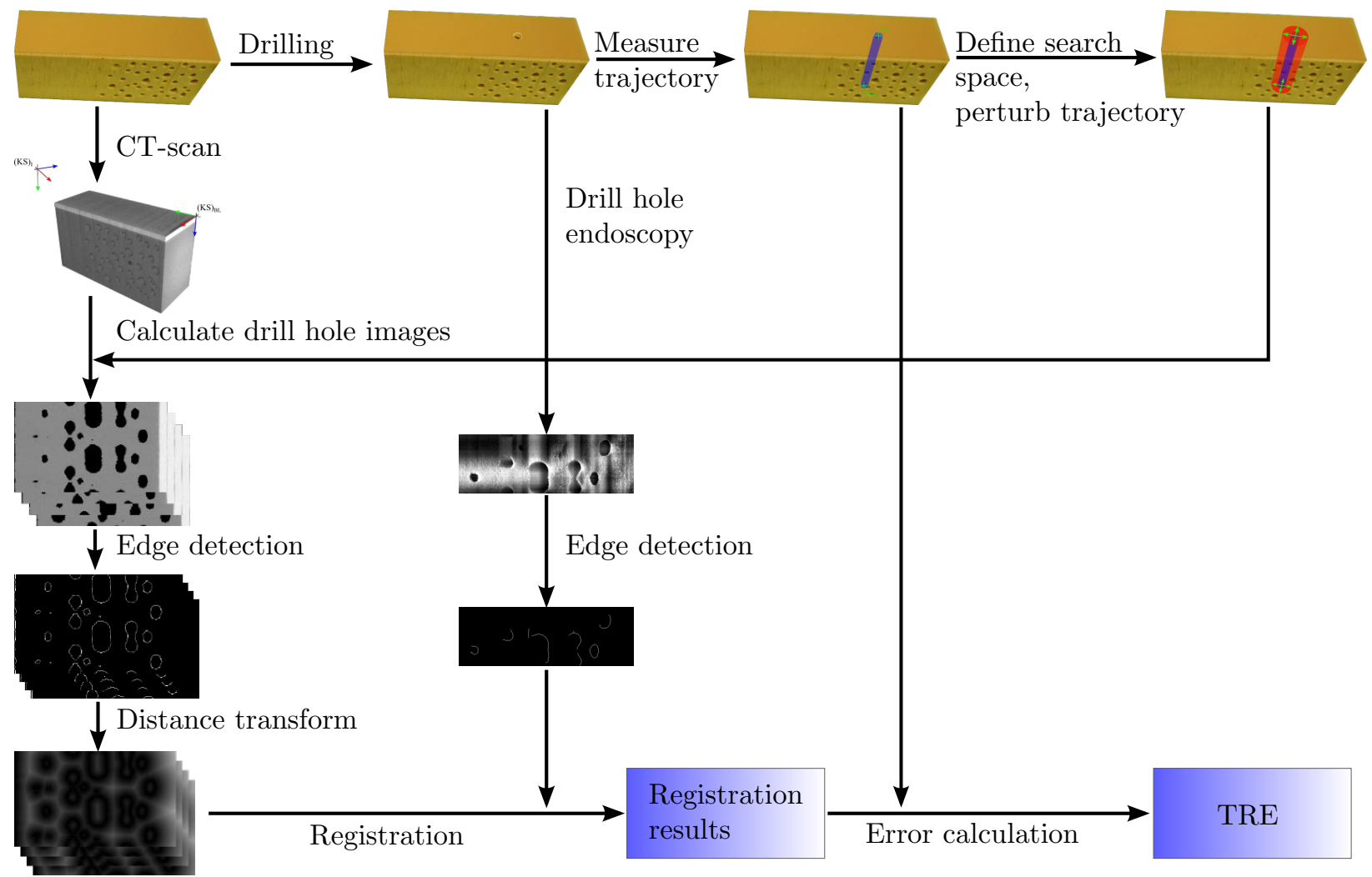

Figure 6. Workflow of the registration procedure, using chamfer matching for image-to-image registrations.

(Microsoft Corporation, Redmond, WA, USA). Sidewalls of the mastoid cavities are only visible in axial advance direction but not in the opposite direction, producing anisotropic imaging characteristics. This effect is exemplary marked with red and green arrows. Red arrows mark the back wall or inside views into mastoid cavities while green arrows mark the front edges of the cavities against the viewing direction.

The image in Fig. 7 b) shows a similar inner drill hole surface, recorded by employing our cone mirror assembly. After unrolling, spatially connected image frames are stitched to create a larger image. Small strips were directly appended without blending (see Sec. 2.4). In comparison to the $0^{\circ}$ optics, a better view into the cavities is provided. Due to the perpendicular imaging, no anisotropic effects relative to the insertion direction are noticed.

a)

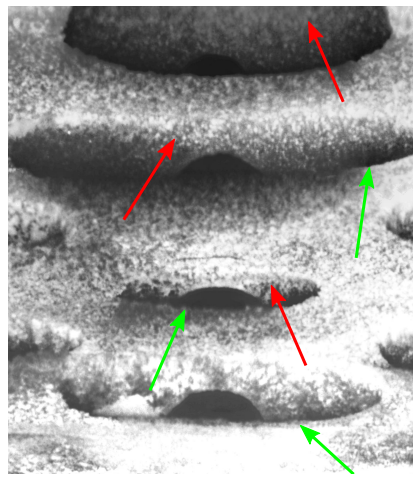

b)

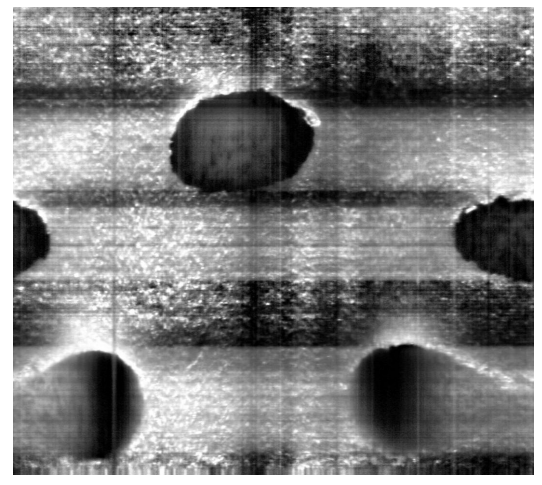

c)

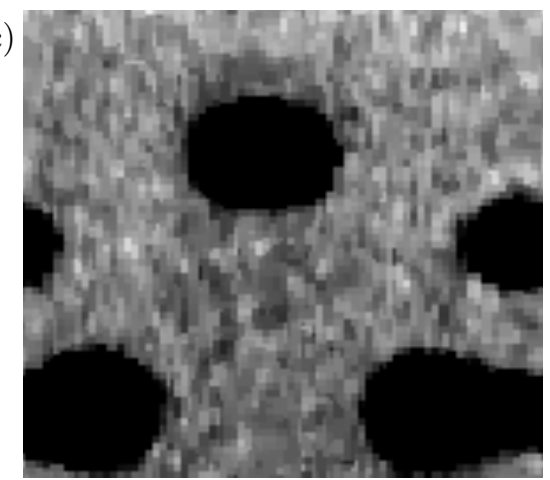

Figure 7. Unrolled and stitched image data, showing the drill hole surface. Left: Recording, using a standard $0^{\circ}$ Storz Hopkins II endoscope optics. Red arrows exemplary mark back walls and green arrows front edges of mastoid cavities. Center: Recording, using our newly developed cone mirror optics. Right: Unrolled surface, extracted from CT image data with voxel size of $0.16 \mathrm{~mm}^{3}$. 
Illumination inhomogeneities occur due to reflections of the provided light at the edges of the simulated mastoid cells. Thus, image strips near these cavities appear brighter. Therefore, a registration approach based on the edges of the visible mastoid cells has been chosen for image-to-image comparison.

The unrolled surface from a simulated drill hole in volumetric image data (CBCT) in Fig. 7 c) shows no background at all in the mastoid cells, since only a single layer at the drill hole surface is unrolled. Artificial mastoid cavities are clearly distinguishable from the bone substitute. Resolution is significantly lower in comparison to the endoscopic images, due to the voxel size of the originating image data of $0.16 \mathrm{~mm}^{3}$. Inhomogeneities in illumination emerge from inhomogeneous material of the test block.

An unrolled image of the drill hole surface from endoscopic recordings and an exemplary image created from $\mathrm{CT}$ image data, which are applied to the registration are shown in Fig. 8 a) and b), respective. The endoscopic image contains all available mastoid cells that are visible over the drill hole length while the simulated images from CT data have the full length of the test block. Overlay visualizations of exemplary image-to-image registration results are presented in Fig. $8 \mathrm{c}$ ). Detected edges are shown in the best registered position for comparison of the endoscopic image to an unrolled CT image. Contours of the CT image are presented in green, contours of the endoscopic image in magenta. The values under the images give the registration metric results for each image. These images provide a visually comprehensible representation of the results. Overlapping contours can be compared and set into relation to the metric values.

a)

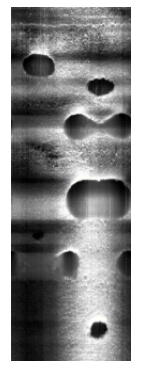

b)

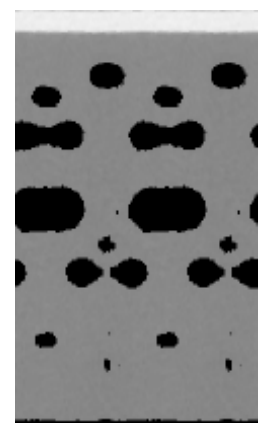

c)

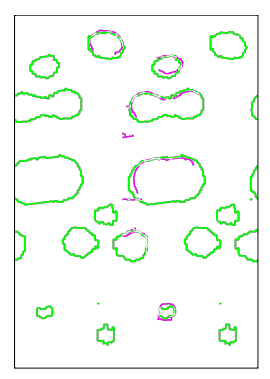

4.378

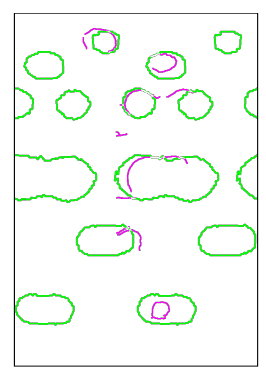

10.763

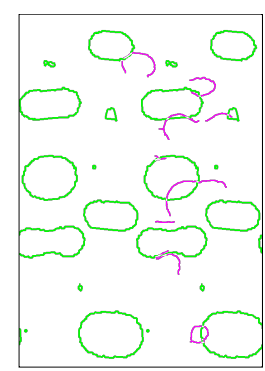

15.999

Figure 8. Image-to-image registration results. a) Stitched image from endoscopic image data. b) Exemplary image of double unrolled drill hole surface from CT image data. c) Comparison of three image-to-image comparisons as overlay images. The edges of the endoscopic images (magenta) are superimposed onto the ones of the CT-images (green). The corresponding registration value of the chamfer matching metric is given under the pictures. Lower values means a better result.

Table 2 gives the results of the regstration accuracy for all ten perturbed search spaces as described in Sec. 2.5. An accuracy of $(0.487 \pm 0.438) \mathrm{mm}$ at the entry, $(0.620 \pm 0.611) \mathrm{mm}$ at the facial recess and $(0.957 \pm 0.948) \mathrm{mm}$ at the exit are achieved. The angular deviation is $(1.763 \pm 1.536)^{\circ}$. Evaluating the absolute $\mathrm{x}$ - and $\mathrm{y}$-components $|\Delta x|$ and $|\Delta y|$, it can be observed that the y-components of the errors are about one order of magnitude smaller than the x-components. Exemplary at the exit, where the highest error occurs, the $\mathrm{x}$-component is $(0.947 \pm 0.952) \mathrm{mm}$ while the $\mathrm{y}$-component is $(0.088 \pm 0.062) \mathrm{mm}$. The directions of the components in block coordinates can be seen in Fig. 3 a). The biggest error component in y-direction is $0.227 \mathrm{~mm}$ at the exit point of trajectory 2 .

The boxplot in Fig. 9 also shows the results of Table 2. The $\mathrm{x}$ - and $\mathrm{y}$-components are plotted next to the TREs. The plot reveals outliers from trajectories 1 and 9. It also shows the similarity between TRE and $\mathrm{x}$-component, confirming neglectable influence of y-component or rather the high accuracy in y-direction.

\section{CONCLUSION}

As of today, endoscopy is a widespread imaging technique in medical applications. Miniaturization opened these techniques to more and more fields of application, e.g. ENT or Neurosurgery. Besides conventional straightand oblique-viewing endoscopes, panoramic and radial imaging devices have been developed. However, these panoramic imaging techniques have not yet been miniaturized to our size requirements. In this publication, 
Table 2. TREs in $\mathrm{mm}$ at critical points along the drill trajectory for ten trajectories and the angular deviations. For each point, the absolute errors in $\mathrm{x}$ - and $\mathrm{y}$-direction in $\mathrm{KS}_{\mathrm{Bl}}$ (also in $\mathrm{mm}$ ) are given.

\begin{tabular}{c|ccc|ccc|ccc|c}
\hline Trajectory & \multicolumn{4}{|c|}{ Entry } & \multicolumn{3}{c|}{ Facial recess } & \multicolumn{5}{c|}{ Exit } & \\
& TRE & $|\Delta x|$ & $|\Delta y|$ & TRE & $|\Delta x|$ & $|\Delta y|$ & TRE & $|\Delta x|$ & $|\Delta y|$ & $\Delta \theta$ \\
\hline 1 & 0.024 & 0.009 & 0.022 & 2.368 & 2.365 & 0.057 & 3.379 & 3.378 & 0.090 & $4.507^{\circ}$ \\
2 & 0.190 & 0.190 & 0.008 & 0.419 & 0.386 & 0.161 & 0.671 & 0.631 & 0.227 & $1.138^{\circ}$ \\
3 & 0.343 & 0.343 & 0.019 & 0.348 & 0.337 & 0.086 & 0.636 & 0.625 & 0.115 & $1.304^{\circ}$ \\
4 & 0.727 & 0.725 & 0.064 & 0.444 & 0.443 & 0.018 & 0.939 & 0.939 & 0.001 & $2.233^{\circ}$ \\
5 & 0.492 & 0.492 & 0.000 & 0.553 & 0.553 & 0.017 & 0.997 & 0.997 & 0.023 & $1.995^{\circ}$ \\
6 & 0.193 & 0.191 & 0.028 & 0.251 & 0.250 & 0.029 & 0.280 & 0.275 & 0.053 & $0.157^{\circ}$ \\
7 & 0.356 & 0.335 & 0.121 & 0.186 & 0.170 & 0.074 & 0.114 & 0.100 & 0.054 & $0.327^{\circ}$ \\
8 & 0.096 & 0.093 & 0.022 & 0.307 & 0.296 & 0.079 & 0.401 & 0.382 & 0.122 & $0.433^{\circ}$ \\
9 & 1.531 & 1.531 & 0.050 & 0.892 & 0.877 & 0.112 & 1.919 & 1.914 & 0.139 & $4.605^{\circ}$ \\
10 & 0.920 & 0.917 & 0.069 & 0.434 & 0.430 & 0.061 & 0.231 & 0.223 & 0.057 & $0.931^{\circ}$ \\
\hline Mean & 0.487 & 0.482 & 0.040 & 0.620 & 0.611 & 0.069 & 0.957 & 0.947 & 0.088 & $1.763^{\circ}$ \\
Std & 0.438 & 0.440 & 0.035 & 0.611 & 0.613 & 0.042 & 0.948 & 0.952 & 0.062 & $1.536^{\circ}$ \\
\hline
\end{tabular}

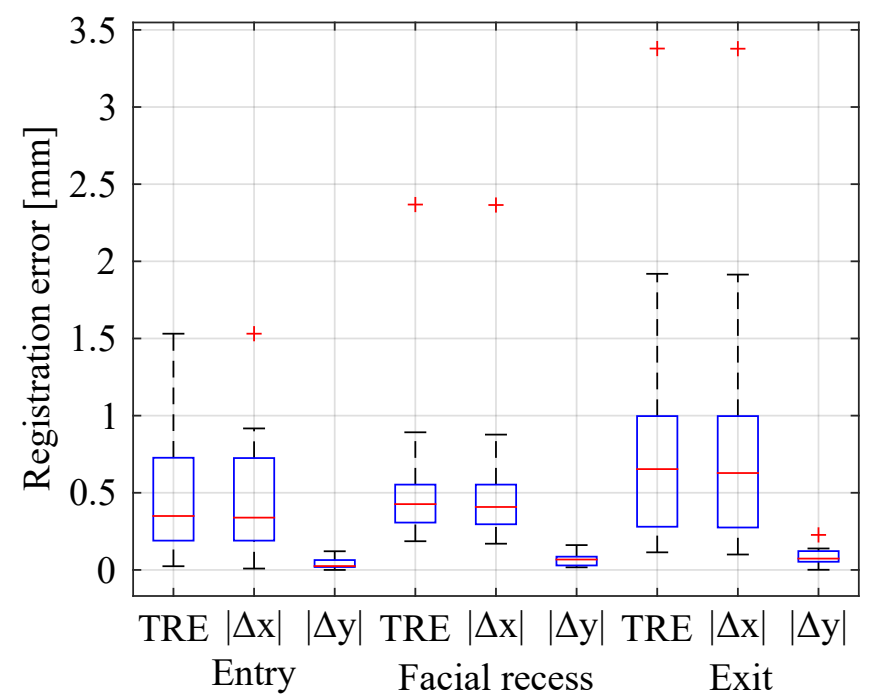

Figure 9. Boxplot, showing registration results of Table 2. 
we presented the first imaging device with a diameter of $3 \mathrm{~mm}$ that is able to record radial $360^{\circ}$ images using a one-shot procedure. Image processing techniques for unrolling and stitching of endoscopic video frames were developed and used to create unrolled panorama images of the inner drill hole surface. These images were applied to a registration of a drill hole inside a test block with artificial mastoid cells.

Registration errors as well as the angular deviations were evaluated at several points along the drill trajectories. Mean TRE errors were below $1 \mathrm{~mm}$ for all points along the drill trajectory while outliers are present. By dividing the TREs into the components along and orthogonal to the mastoid drill holes, influences of the pattern variation could be distinguished. Error components in the direction of the mastoid drill holes (x-direction) were about ten times higher than in the direction orthogonal to the mastoid (y-direction).

Since the mastoid drill holes are through holes, they do not vary in drilling direction, meaning the drill pattern is not variant in all directions. In direction of the mastoid drill holes (x-direction), a translation of the drill hole means no change of the visible pattern and results therefore into high registration errors. Also, tilting of the drill hole in this direction creates only small variations of the drill pattern, making these difficult to distinguish. In contrast, the small errors of the y-components are significantly lower and show the potential of the method. In this direction, the mastoid pattern varies with translation and tilt of the drill hole, leading to good distinguishable patterns at the inner drill hole surface.

\section{ACKNOWLEDGMENTS}

This work has been supported by the Deutsche Forschungsgemeinschaft (DFG) grant KA 2975/4-1.

\section{REFERENCES}

[1] Bergmeier, J., Daentzer, D., Noll, C., Majdani, O., Ortmaier, T., and Kahrs, L. A., "Towards endoscopic image-to-physical registration of mastoid cells and trabecula," Annu. Conf. Ger. Soc. Comput. Robot Assist. Surg., 43-48 (2015).

[2] Bergmeier, J., Daentzer, D., Majdani, O., Ortmaier, T., and Kahrs, L. A., "Image-to-physical registration based on endoscopy of a drill hole inside bone," CARS 2016-Computer Assist. Radiol. Surg. Proc. 30th Int. Congr. Exhib. Heidelb., 206-207 (2016).

[3] Bell, B., Williamson, T., Gerber, N., Gavaghan, K., Wimmer, W., Kompis, M., Weber, S., and Caversaccio, M., "An image-guided robot system for direct cochlear access.," Cochlear Implants Int. 15 Suppl 1, S11-3 (2014).

[4] Labadie, R. F., Balachandran, R., Noble, J. H., Blachon, G. S., Mitchell, J. E., Reda, F. A., Dawant, B. M., and Fitzpatrick, J. M., "Minimally invasive image-guided cochlear implantation surgery: First report of clinical implementation," Laryngoscope 124(8), 1915-1922 (2014).

[5] Majdani, O., Rau, T. S., Baron, S., Eilers, H., Baier, C., Heimann, B., Ortmaier, T., Bartling, S., Lenarz, T., and Leinung, M., "A robot-guided minimally invasive approach for cochlear implant surgery: Preliminary results of a temporal bone study," Int. J. Comput. Assist. Radiol. Surg. 4(5), 475-486 (2009).

[6] Williamson, T. M., Bell, B. J., Gerber, N., Salas, L., Zysset, P., Caversaccio, M., and Weber, S., "Estimation of tool pose based on force-density correlation during robotic drilling," IEEE Trans. Biomed. Eng. 60(4), 969-976 (2013).

[7] Bergen, T. and Wittenberg, T. M., "Stitching and Surface Reconstruction from Endoscopic Image Sequences: A Review of Applications and Methods.," IEEE J. Biomed. Heal. informatics 2194(c), 1-20 (2014).

[8] Richard Wolf GmbH, "Technoskop mit Rundumausblick," (1979).

[9] Ou-Yang, M. and Jeng, W.-D., "Design and analysis of radial imaging capsule endoscope (RICE) system.," Opt. Express 19(5), 4369-4383 (2011).

[10] Jeng, W.-D., Mang, O.-Y., Chen, Y.-T., and Wu, Y.-Y., "Design of illumination system in ring field capsule endoscope," Proc. SPIE 7893, 78930E-78930E-9 (2011).

[11] Spacek, L., "A catadioptric sensor with multiple viewpoints," Rob. Auton. Syst. 51(1), 3-15 (2005).

[12] Kobler, J.-P., Schoppe, M., Lexow, J. G., Rau, T. S., Majdani, O., Kahrs, L. A., and Ortmaier, T., "Temporal Bone Borehole Accuracy for Cochlear Implantation Influenced by Drilling Strategy: an In Vitro Study," Int. J. Comput. Assist. Radiol. Surg. 9, 1033-1043 (2014). 
[13] Liu, M. Y., Tuzel, O., Veeraraghavan, A., and Chellappa, R., "Fast directional chamfer matching," Proc. IEEE Comput. Soc. Conf. Comput. Vis. Pattern Recognit. , 1696-1703 (2010).

[14] Maurer, C. and Raghavan, V., "A linear time algorithm for computing exact Euclidean distance transforms of binary images in arbitrary dimensions," IEEE Trans. Pattern Anal. Mach. Intell. 25(2), 265-270 (2003). 